\title{
Interactive Classification of Remote Sensing Images by using Optimum-Path Forest and Genetic Programming
}

\author{
Jefersson Alex dos Santos ${ }^{1}$, André T. da Silva ${ }^{2}$, Ricardo da S. Torres ${ }^{1}$, \\ Alexandre X. Falcão ${ }^{1}$, Léo P. Magalhães ${ }^{2}$, and Rubens A. C. Lamparelli ${ }^{3}$ \\ 1 Institute of Computing \\ 2 School of Electrical and Computer Engineering \\ 3 Center for Research in Agriculture \\ University of Campinas, Campinas, SP, Brazil \\ jsantos@ic.unicamp.br, atavares@dca.fee.unicamp.br, rtorres@ic.unicamp.br, \\ afalcao@ic.unicamp.br, leopini@fee.unicamp.br, rubens@cpa.unicamp.br,
}

\begin{abstract}
The use of remote sensing images as a source of information in agribusiness applications is very common. In those applications, it is fundamental to know how the space occupation is. However, identification and recognition of crop regions in remote sensing images are not trivial tasks yet. Although there are automatic methods proposed to that, users very often prefer to identify regions manually. That happens because these methods are usually developed to solve specific problems, or, when they are of general purpose, they do not yield satisfying results. This work presents a new interactive approach based on relevance feedback to recognize regions of remote sensing. Relevance feedback is a technique used in content-based image retrieval (CBIR) tasks. Its objective is to aggregate user preferences to the search process. The proposed solution combines the Optimum-Path Forest (OPF) classifier with composite descriptors obtained by a Genetic Programming (GP) framework. The new approach has presented good results with respect to the identification of pasture and coffee crops, overcoming the results obtained by a recently proposed method and the traditional Maximimun Likelihood algorithm.
\end{abstract}

Keywords: Remote Sensing Image Classification; Genetic Programming; Optimum-Path Forest; Relevance Feedback

\section{Introduction}

Agriculture productivity is strongly dependent on monitoring and planning activities. Production estimation and land use are the basis for government policies to finance agricultural activities. Thus, there is a huge demand for information systems that allow to store, analyze, and handle geographic data. This is the purpose of Geographic Information Systems (GISs). Most of existing GIS-based applications rely on the use of Remote Sensing Images (RSIs) to crop monitoring. 
Because RSIs are raster data, to obtain vectorial information is necessary to extract regions of interest. In addition to the typical problems in pattern recognition research, identifying crop areas in RSIs faces hard problems associated, for instance, with terrain distortions. Besides that, RSIs, contrary to common images, do not encode just human visible information, but also other spectral bands (for example, infrared). For this reason, the recognition task normally needs classification strategies which exploit RSI properties related to both spectral and texture patterns.

The process of recognizing regions is called classification and can be done both automatically or manually. Sometimes users prefer to identify regions manually because the results of automatic approaches are unsatisfactory. The most successful RSI classification methods are normally created to a specific target or data [14]. General purpose methods, however, are very sensitive to noise. Furthermore, the spectral response and the texture patterns observed for a given crop can be different. A crop can be planted in different ways and this factor, allied to the different phases of plants, tends to create distinction between regions where the same culture is being cultivated. Therefore, in practical situations, the results of automatic methods need to be revised.

This work aims to present a new interactive approach for classifying regions in RSIs. The proposed solution relies on the use of an interactive strategy, called relevance feedback $(\mathrm{RF})$, based on which the classification system can learn what regions are of interest, given what is indicated by users. The proposal is a new hybrid method, named GOPF, which uses a GP framework to create composite image descriptors [5] and the optimum-path forest (OPF) classifier [11] to determine regions of interest. OPF is a classification method which represents each class of objects by one or multiple optimum-path trees rooted at key samples, called prototypes.

\section{Related Work}

In [9], Lu \& Weng present an overview of the problem of classification of remote sensing images including the steps which comprise the process (extraction of features, segmentation, classification and accuracy assessment) and the research challenges faced. For each step, most of the existing techniques until 2005 are presented, grouped by the approach adopted (such as techniques that exploits classification by pixels or regions).

The classification algorithm based on pixels, MaxVer (Maximum Likelihood Classification) [12], is still one of the most popular. On the other hand, the growth of classification approaches based on regions, for instance, is analyzed in [3]. The article proves that the growth in the number of new approaches published accompanies the increase of the accessibility to high-resolution images and, hence, the development of alternative techniques to the classification based on pixels.

Apart from the classification methods presented in [14,12,3], several others have been proposed recently $[8,13,1,10,2]$. The novelty of these approaches re- 
lies on: resolution and number of bands of ISRs; type of extracted features; used learning technique, and level of discrimination among the classes of the image (some studies include all the vegetation types in the same class, for example). Li et. al [8] proposed a method based on a regions adjacency graph for segmentation of images with high resolution. The regions are segmented according to the combination of shape, color and texture features. The RSIs classification methods proposed by Munoz-Mari et al. [10] and Basi \& Melgani [2] are based on Support vector machines (SVMs). The first [10] proposes a supervised classification method called SVDD. The experiments were made by differentiating various classes of vegetation (corn, grass, pasture, trees, etc.). As far as [2] is concerned, they proposed an RSIs classification system based on SVM in which Genetic Algorithms (GA) are used to find the best set of parameters of SVM. The extracted features are based on pixels. Low-resolution aerial images were used. Besides [2], both RSIs classification methods proposed by Tseng et. al [13] and Bandyopadhyay et. al [1] use Genetic algorithms (GA). The former uses GA to find the configuration parameters of a neural network while the latter uses GA for clustering the pixels of the RSIs. Although both use the pixel information, in [13], the vegetation classes are distinguished and images of middle and high resolution are used.

The main advantages of the proposed method against the aforementioned approaches are the use of a runtime technique for combination of features (genetic programming) and the refinement of the OPF-based classification system by taking into account the user interaction. Moreover, most of the mentioned works do not address the problem of specific crops recognition. They group different classes into larger sets or even into a single one. We have recently proposed an interactive method for classification of remote sensing images based on Genetic Programming, $G P_{S R}$ [6]. That method allows users to interact with the classification system, indicating regions of interest (and those which are not). This feedback information is employed by a genetic programming approach for learning user preferences and combining image region descriptors that encode spectral and texture properties. One remarkable advantage of the proposed method when compared with $G P_{S R}$ is that it does not need thresholds for selecting seeds to be used in the segmentation process. At the end of each relevance feedback interaction, the classifier itself defines the relevance level for all of the subimages. $G P_{S R}$ is used as baseline in our experiments.

\section{The GOPF Approach}

The GOPF approach is a framework for recognition of regions of interest in remote sensing images combining $O P F$ [11] classifier and GP [5] composite descriptor. We also adopt the definition of simple descriptor used in [5], which is composed by a pair consisting of an extraction function and a distance function.

Optimum-path forest (OPF) is a classification method that represents each class of objects by one or multiple optimum-path trees rooted at key samples, called prototypes [11]. The training samples are nodes of a complete graph. In our 
work, the arcs are weighted by the distance provided by the composite descriptor of their nodes. The use of OPF for relevance feedback considers two classes: relevant subimages (chosen by the user) and irrelevant ones. The prototypes computed by the OPF classifier are used to rank database images according to the user's selection.

Algorithm 1 illustrates how GOPF is used in the classification system. Let $\hat{I}$ be an RSI divided into a set of subimages (block regions). The distance $d(s, t)$ between two subimages $s$ and $t$ is the distance between their corresponding feature vectors combined by a function (composite descriptor). For an initial query point $s$, the proposed method returns the $N$ closest subimages in $\hat{I}$ to $s$ (query by similarity). Due to the semantic gap problem, the closest subimages to $s$ may not be the most relevant for a given user. By marking the relevant subimages among the returned ones, the user creates two sets: a set $\mathcal{I} \subset \hat{I}$ of irrelevant subimages and a set $\mathcal{R} \subset \hat{I}$ of relevant subimages. The method then uses sets $\mathrm{R}$ and I to compute relevant (set A) and irrelevant (set B) sets of prototypes and two optimum-path forests rooted at them. Each subimage $t \in \hat{I} \backslash \mathcal{I} \cup \mathcal{R}$ is then classified according to the root's label of the forest (relevant/irrelevant) that offers to t the optimum path from $\mathcal{A} \cup \mathcal{B}$. Only the $N$ closest images labeled as relevant will be returned (in a set $\mathcal{C}$ ) to the user in the next iteration. Relevant prototypes $(\mathcal{A})$ and irrelevant ones $(\mathcal{B})$, computed in the previous step, are then used to sort the subimages in $\mathcal{C}$ for the next iteration. The method computes the average distance $\bar{d}_{\mathcal{A}}(t, \mathcal{A})$ between each subimage $t \in \mathcal{C}$ and subimages in the set of relevant prototypes $\mathcal{A}$. It also computes the average distance $\bar{d}_{\mathcal{B}}(t, \mathcal{B})$ between $t$ and subimages in the set of irrelevant prototypes $\mathcal{B}$. Finally, a distance $\bar{d}(t, \mathcal{A}, \mathcal{B})$ is computed as a normalized mean between relevant and irrelevant prototypes according to the composite descriptor: $\bar{d}(t, \mathcal{A}, \mathcal{B})=\frac{\bar{d}_{\mathcal{A}}(t, \mathcal{A})}{\bar{d}_{\mathcal{A}}(t, \mathcal{A})+\bar{d}_{\mathcal{B}}(t, \mathcal{B})}$. After classifying each subimage in $\hat{I} \backslash \mathcal{I} \cup \mathcal{R}$, the method returns to the user a new set of $N$ relevant subimages, which contains the lowest values of $\bar{d}(t, \mathcal{A}, \mathcal{B})$ This process is then repeated for a few iterations $T$ and, finally, the system returns all relevant subimages obtained so far. The complete approach, which we call GOPF $(\mathrm{GP}+\mathrm{OPF})$, is illustrated in Figure 1 (a).

The performance of the classifier is directly dependent on the good description of the objects involved in the classification. In order to combine spectral and texture distances from various descriptors, the distance $d(s, t)$ between two images $s$ and $t$ used by OPF classifier is provided by a GP-based composite descriptor [5]. The GP framework requires a training set to find a good combination function. As the method is interactive we propose training GP with the prototypes $(\mathcal{A}$ and $\mathcal{B})$ provided by the OPF since they are very informative subimages. The GP module starts with a population of combination functions created randomly. This population evolves generation by generation through genetic operations (e.g., crossover, mutation, reproduction). A fitness function is used to assign the fitness value for each individual based on the ranking of the training set. This value is used to select the best individuals. Next, genetic operators are applied to this population aiming to create more diverse and better 


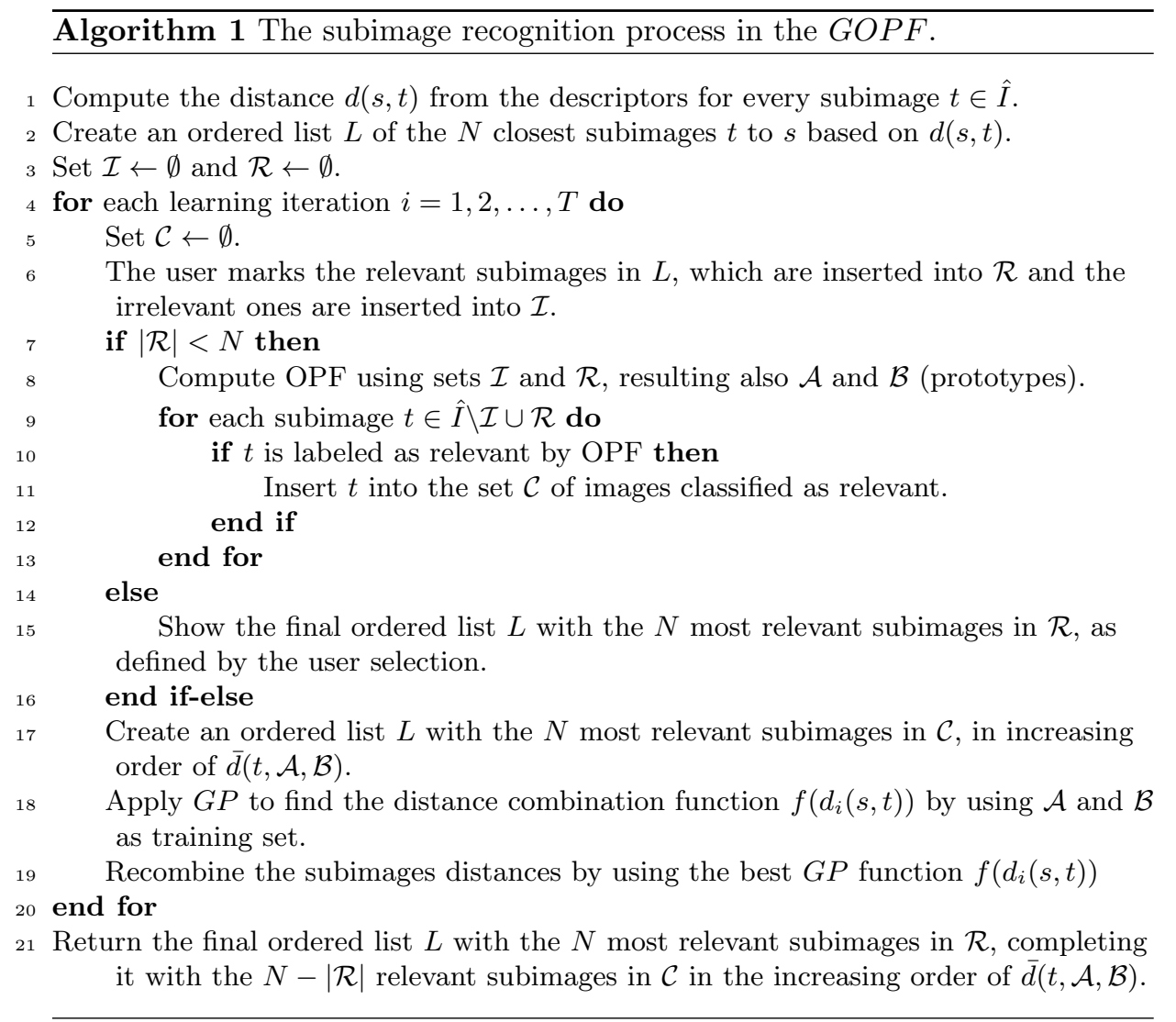

performing individuals. The last step consists in determining the best individual to be applied to the test set. The commonest choice is the individual with the best performance in the training set (e.g., the first function of the last generation).

In this work we adopt the same notion of descriptor proposed in [5]. In this case, a GP individual is a function used to combine the distances provided by a set of single descriptors concerning the features extracted from two subimages. The GP individual configuration in the GOPF is the same as that used for $G P_{S R}$ method [6]. Figure 1 (b) shows an example of GP individual as a function to combine descriptors from two subimages. This individual corresponds to the function $f\left(d_{1}(s, t), d_{2}(s, t), d_{3}(s, t)\right)=\frac{d_{1}(s, t) * d_{3}(s, t)}{d_{2}(s, t)}-\sqrt{d_{2}(s, t)+d_{3}(s, t)}$.

\section{Experiments}

This section describes the experiments performed to validate our method. The experiment Setup is described as the following: 


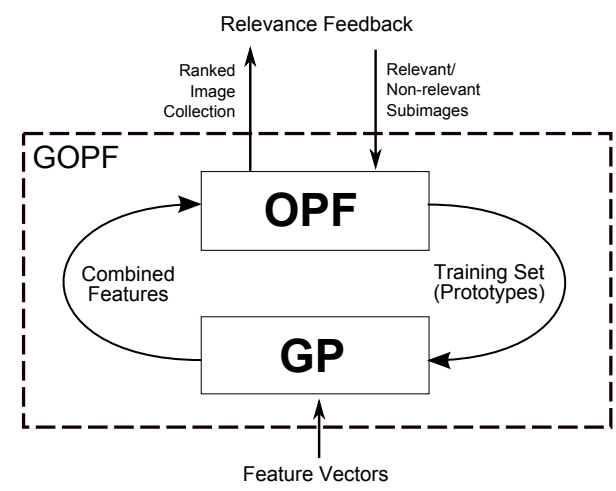

(a)

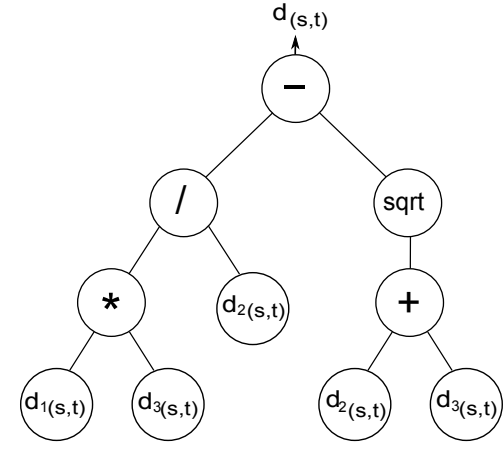

(b)

Fig. 1. The proposed interactive classifier (GOPF).

GP Parameters: Population size was 60; the number of generations, 10; initial population, half-and-half; initial tree depth, between 2 and 5; maximum tree depth, 5; selection method, tournament with size 2; crossover rate, 0.8 ; mutation rate, 0.2 ; and functions set is composed by the operators $+, *, \sqrt{ }, d^{\text {const }}$. Descriptors: We used the same set of descriptors in [6]: BIC, Color Histograms, Color Moments, Gabor Wavelets, and Spline Wavelets. Baseline: We compare our method against Maximum Likelihood Classification (MaxVer) [12] and $G P_{S R}[6]$. PASTURE image was classified by MaxVer with probability threshold 0.8 and using 20,580 points of pasture sample. COFFEE image was classified with probability threshold 0.98 and using 43,630 points of coffee sample. Effectiveness measure: We use kappa-iterations curves as effectiveness measure. Kappa [4] is an effective index to compare classified images, commonly used in RSI classification. Experiments in different areas show that kappa could have various interpretations and these guidelines could be different depending on the application. However, Landis and Koch [7] characterize Kappa values over 0.80 as "almost perfect agreement", 0.60 to 0.79 as "substantial agreement", 0.40 to 0.59 as "moderate agreement", and below 0.40 as "poor agreement". Kappa negative means that there is no agreement between classified and verification data. Data: Two RSIs were used to validade our method. Information about used RSIs is showed in Table 1. In this paper, we call Image 1 PASTURE and image 2 COFFEE. These images were divided into subimages of $20 \times 20$ and $30 \times 30$ pixels, respectively. Thus, the image PASTURE is composed by 5900 while COFFEE is composed by 6400 subimages. A total of 100 different subimages were used as initial query point $s$. The results presented are the average for each inital pattern $s$.

Figure 2 (a) illustrates the curve kappa-iterations of the GOPF in comparison with $G P_{S R}$ and the value found by the MaxVer for the PASTURE image. Note that the value of kappa is always greater for the proposed method 
Table 1. Remote Sensing Images used in the experiments.

\begin{tabular}{|l|l|l|}
\hline & Image 1 & Image 2 \\
\hline \hline Region of interest & pasture & coffee \\
\hline Terrain & plain & mountainous \\
\hline Satellite & CBERS & SPOT \\
\hline Spatial resolution & 20 meters & 2.5 meters \\
\hline Bands composition & R-IR-G $(342)$ & IR-NIR-R $(342)$ \\
\hline Acquisition date & $08-20-2005$ & $08-29-2005$ \\
\hline Location & Laranja Azeda Basin, MS & Monte Santo County, MG \\
\hline Dimensions $(\mathbf{p x})$ & $1310 \times 1842$ & $2400 \times 2400$ \\
\hline
\end{tabular}

when compared to MaxVer, along iterations. Note also that the hybrid approach, $G O P F$, yields better results than $G P_{S R}$.

Figure 2 (b) illustrates the curve-kappa iterations of $G O P F$ in comparison with the kappa value found by $G P_{S R}$ and MaxVer for the COFFEE image. The kappa values for the proposed methods were better than the MaxVer score. Another remarkable result is concerned with the superiority of GOPF when compared to $G P_{S R}$.

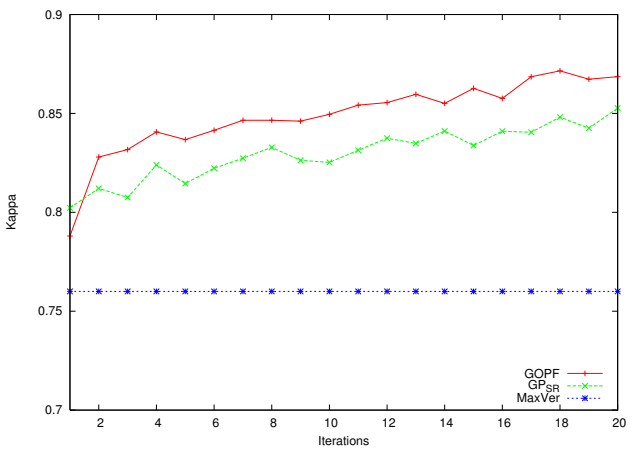

(a)

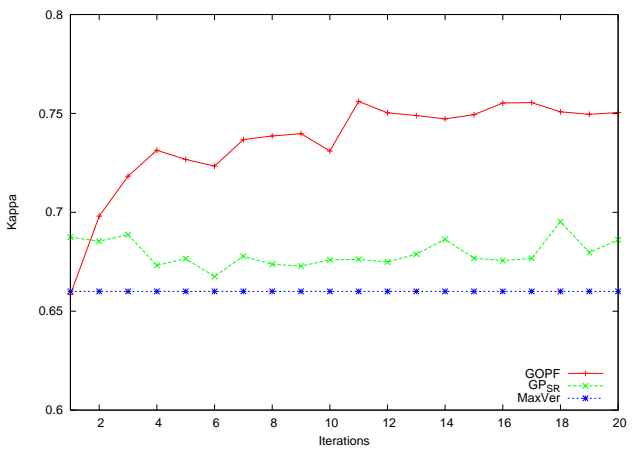

(b)

Fig. 2. Kappa X Iterations comparing the results of the proposed method, $G P_{S R}$ and the MaxVer to the PASTURE image (a) and to the COFFEE image.

\section{Conclusions}

We have proposed a hybrid framework for recognition of regions of interest in remote sensing images which combines $O P F$ [11] classifier and $G P$ [5] composite descriptor. The system uses image descriptors to encode the spectral and texture regions of the RSIs and exploits user's relevance feedback. GOPF has presented good results with respect to the identification of pasture and coffee 
crops, overcoming the results obtained by $G P_{S R}[6]$ and the MaxVer algorithm. As future works, we plan to evaluate more image descriptors; to allow user to define multiple regions as query pattern; and to compare the method with other baselines.

\section{Acknowledgments}

The authors are grateful to CAPES, FAPESP, and CNPq for financial support.

\section{References}

1. S. Bandyopadhyay, U. Maulik, and A. Mukhopadhyay. Multiobjective Genetic Clustering for Pixel Classification in Remote Sensing Imagery. IEEE Transactions on Geoscience and Remote Sensing, 45:1506-1511, May 2007.

2. Bazi and Melgani. Toward an Optimal SVM Classification System for Hyperspectral Remote Sensing Images. IEEE Transactions on Geoscience and Remote Sensing, 44:3374-3385, November 2006.

3. T. Blaschke. Object based image analysis for remote sensing. ISPRS Journal of Photogrammetry and Remote Sensing, 65(1):2 - 16, 2010.

4. Russel G. Congalton and Kass Green. Assessing the Accuracy of Remotely Sensed Data: Principles and Practices. Lewis Publishersr, Washington, DC, 1977.

5. R. da S. Torres, A.X. Falcão, M.A. Gonçalves, J.P. Papa, B. Zhang, W. Fan, and E.A. Fox. A genetic programming framework for content-based image retrieval. Pattern Recognition, 42(2):217-312, Feb 2009.

6. J.A. dos Santos, C.D. Ferreira, R. da S.Torres, M.A. Gonçalves, and R.A.C. Lamparelli. A relevance feedback method based on genetic programming for classification of remote sensing images. Information Sciences, 181(13):2671 - 2684, 2011.

7. J. R. Landis and G. G. Koch. The measurement of observer agreement for categorical data. Biometrics, 33(1):159-174, March 1977.

8. N. Li, H. Huo, and T. Fang. A novel texture-preceded segmentation algorithm for high-resolution imagery. Geoscience and Remote Sensing, IEEE Transactions on, (99):1 -11, 2010.

9. D. Lu and Q. Weng. A survey of image classification methods and techniques for improving classification performance. International Journal of Remote Sensing, 28(5):823-870, 2007.

10. J. Munoz-Mari, L. Bruzzone, and G. Camps-Valls. A Support Vector Domain Description Approach to Supervised Classification of Remote Sensing Images. IEEE Transactions on Geoscience and Remote Sensing, 45:2683-2692, August 2007.

11. J.P. Papa, A.X. Falcão, and C.T.N. Suzuki. Supervised pattern classification based on optimum-path forest. International Journal of Imaging Systems and Technology, 19(2):120-131, 2009.

12. Robert Showengerdt. Techniques for Image Processing and Classification in Remote Sensing. Academic Press, New York, 1983.

13. Ming-Hseng Tseng, Sheng-Jhe Chen, Gwo-Haur Hwang, and Ming-Yu Shen. A genetic algorithm rule-based approach for land-cover classification. ISPRS Journal of Photogrammetry and Remote Sensing, 63(2):202 - 212, 2008.

14. G.G. Wilkinson. Results and implications of a study of fifteen years of satellite image classification experiments. IEEE Transactions on Geoscience and Remote Sensing, 43(3):433-440, March 2005. 